\title{
Hypertrophic Cardiomyopathy With Midventricular Obstruction and Apical Aneurysm A Case Report
}

\author{
Kazumasa Harada, MD; Tatsuya Shimizu, MD; Yasuyuki Sugishita, MD; \\ Atsushi Yao, MD; Jun-ichi Suzuki, MD; Katsu Takenaka, MD; \\ Yasunobu Hirata, MD; Ryozo Nagai, MD; Toshiyuki Takahashi, MD
}

\begin{abstract}
A 71-year-old woman had hypertrophic cardiomyopathy associated with midventricular obstruction and an apical aneurysm in the left ventricle. She had had abnormal electrocardiograms for more than 30 years and for the past year had been suffering from occasional attacks of dizziness and low systemic blood pressure. Holter 24-h electrocardiographic monitoring revealed ventricular paroxysmal contractions (676/day) with nonsustained ventricular tachycardia. Doppler echocardiography revealed paradoxical jet flow from the apical aneurysm to the left ventricular outflow during early diastole. Magnetic resonance imaging depicted midventricular hypertrophy and a dyskinetic thin apical wall, which were confirmed by angiography. Coronary angiograms showed no narrowing of the major extramural coronary arteries, but there was compression of aberrant coronary arteries apparently feeding the hypertrophic portion of the left ventricular wall. Stress thallium-201 myocardial imaging showed a persistent severe defect in the left ventricular apex. A hemodynamic study revealed low cardiac output and an intraventricular pressure gradient (approximately $90 \mathrm{mmHg}$ ) between the left ventricular apical high-pressure chamber and the subaortic low-pressure chamber. The present case represents a rare combination of hypertrophic cardiomyopathy, midventricular obstruction, and an apical aneurysm in an elderly woman. Myocardial ischemia may have played an important role in the genesis of the apical aneurysm. (Jpn Circ J 2001; 65: 915-919)
\end{abstract}

Key Words: Apical aneurysm; Hypertrophic cardiomyopathy; Intraventricular pressure gradient; Midventricular hypertrophy

$\mathbf{H}$ ypertrophic cardiomyopathy (HCM) is characterized by an inappropriate left ventricular and/or right ventricular hypertrophy that is often asymmetrical and associated with microscopic evidence of myocardial fiber disarray! ${ }^{1-3}$ Its etiology remains to be elucidated, but it is now recognized that HCM may consist of heterogeneous genetic diseases of the sarcomere. The manifestation of the disease is mostly dependent on the extent and site of hypertrophy and although interventricular septal hypertrophy is by far the most common type, other manifestations, including midventricular, apical, and the rarer types of asymmetrical hypertrophy, have also been described. Midventricular hypertrophy is a rare form of HCM (1\%), which causes midventricular obstruction complicated with apical myocardial infarction in some cases. $^{6-11}$ The involvement of myocardial ischemia has been suggested, but the exact cause of the apical aneurysm remains to be determined. In the majority of the cases, apical infarction is associated with normal extramural coronary arteries, but a 'sawfish' appearance of the coronary arteries has sometimes been reported. We here report a case of HCM with midventricular obstruction, an apical

(Received December 25, 2000; revised manuscript received March 1, 2001; accepted March 15, 2001)

Department of Cardiovascular Medicine, Graduate School of Medicine, University of Tokyo, Japan

Mailing address: Kazumasa Harada, MD, Department of Cardiovascular Medicine, Graduate School of Medicine, University of Tokyo, 7-3-1 Hongo, Bunkyo-ku, Tokyo 113-8655, Japan. E-mail: harada-2im@h.u-tokyo.ac.jp aneurysm, and compression of the aberrant coronary arteries feeding the hypertrophic portion of the left ventricular wall. The etiology of the apical aneurysm and the possible contribution of myocardial ischemia will be discussed.

\section{Case Report}

A 71-year-old woman was admitted for evaluation of a severe intraventricular pressure gradient caused by midventricular obstruction, and an apical aneurysm in the left ventricle. Abnormal ECGs had been recorded more than 30 years before, but for the past year she had been suffering from occasional attacks of dizziness and low systemic blood pressure and HCM was diagnosed on echocardiography. She did not have a family history of heart disease, except for her younger sister who had arrhythmias of unknown origin.

Physical examination revealed a midsystolic murmur and a diastolic murmur of variable intensity (grade 2/6-4/6) along the lower left sternal border. Her blood pressure was $95 / 55 \mathrm{mmHg}$, and the pulse rate was 58 beats $/ \mathrm{min}$. Blood tests showed a decreased platelet count $\left(9.3 \times 10^{4} / \mathrm{ml}\right)$ and elevated serum brain natriuretic peptide $(250 \mathrm{fmol} / \mathrm{ml})$. Anti-hepatitis B virus core antigen antibody was detected, but not anti-hepatitis $\mathrm{C}$ virus antibody. There was elevation of the ST-segment in leads II, III, $\mathrm{aVF}$ and $\mathrm{V}_{2-6}$ and terminal T-wave negativity in the I, II, III, $\mathrm{aVF}_{\mathrm{F}}$, $\mathrm{aVL}$ and $\mathrm{V}_{2-6}$ leads (Fig 1). Holter 24-h electrocardiographic monitoring revealed ventricular paroxysmal contractions (676/day) with nonsustained ventricular 


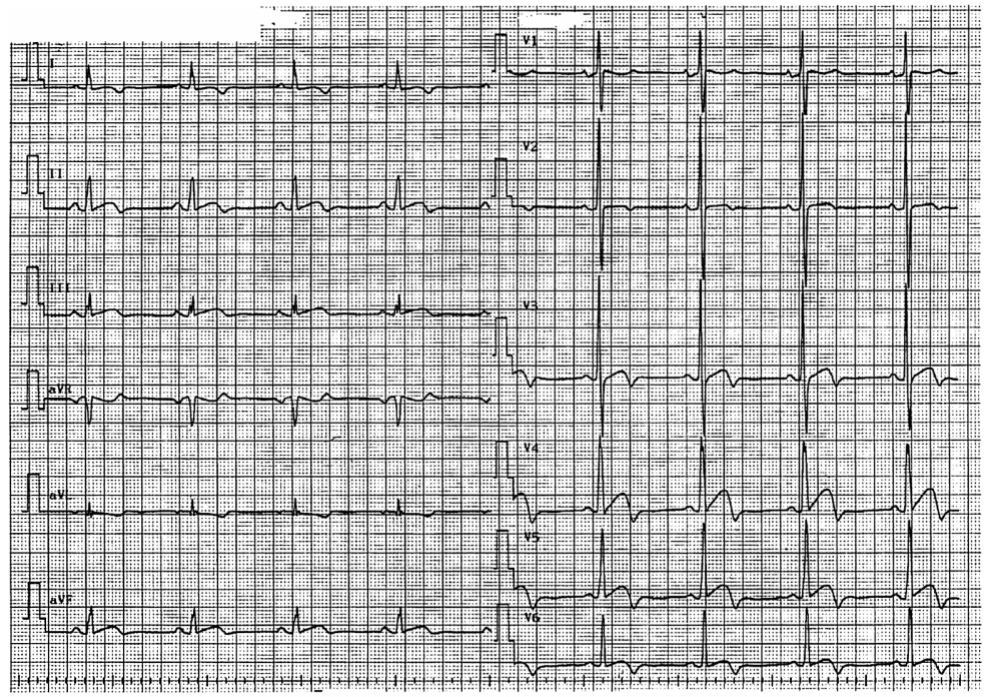

Fig 1. Electrocardiogram.

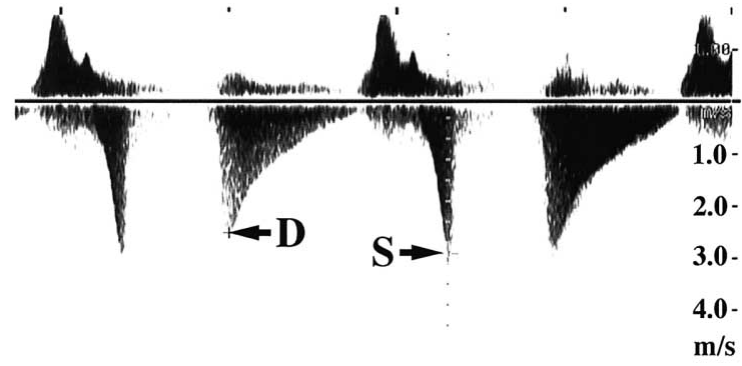

PCG

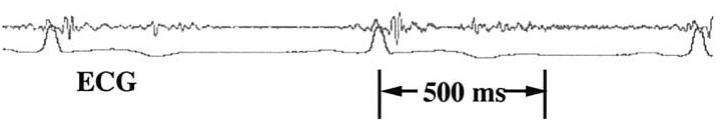

Fig 2. Continuous Doppler ultrasound from the apical window used for assessment of the intraventricular systolic (S) and diastolic (D) peak flow velocities across the obstructed portion. PCG, phonocardiography. tachycardia. Chest X-ray did not show cardiomegaly. Echocardiography revealed asymmetric septal hypertrophy (anteroseptal wall thickness at end-diastolic phase $15 \mathrm{~mm}$, posterior wall thickness $10 \mathrm{~mm}$ ), midventricular obstruction (anteroseptal wall thickness $20 \mathrm{~mm}$ ), and an apical aneurysm (apical wall thickness $6 \mathrm{~mm}$ ), but no evidence of submitral calcification. The left ventricular short axis dimension at end-diastole and at end-systole was $40 \mathrm{~mm}$ and $22 \mathrm{~mm}$, respectively. Doppler echocardiography revealed paradoxical jet flow from the apical aneurysm to the left ventricular outflow during early diastole (Fig 2)!1 Magnetic resonance imaging depicted midventricular hypertrophy and a dyskinetic thin apical wall in the left ventricle (Fig 3).

Cardiac catheterization was performed after admission and the hemodynamic study revealed low cardiac output, normal right atrial pressure (mean $1 \mathrm{mmHg}$ ), no evidence of pulmonary hypertension $(15 / 3 \mathrm{mmHg}$, mean $7 \mathrm{mmHg}$ ), but a peak-to-peak intraventricular pressure gradient of 90 $\mathrm{mmHg}$ during pull-back from the apical high-pressure
A

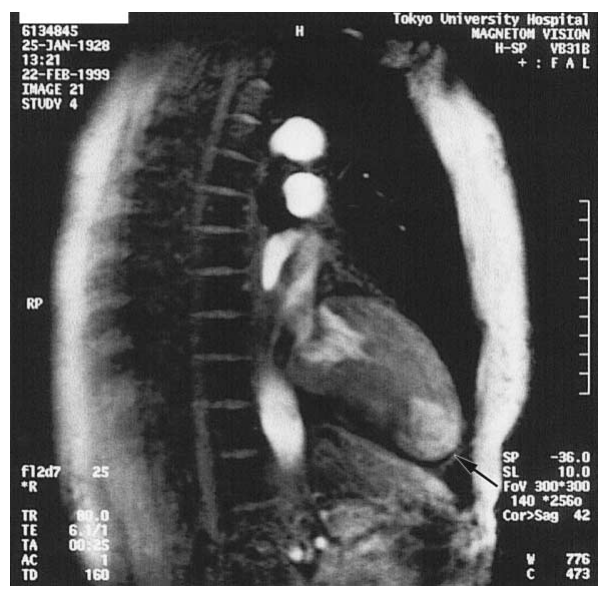

B
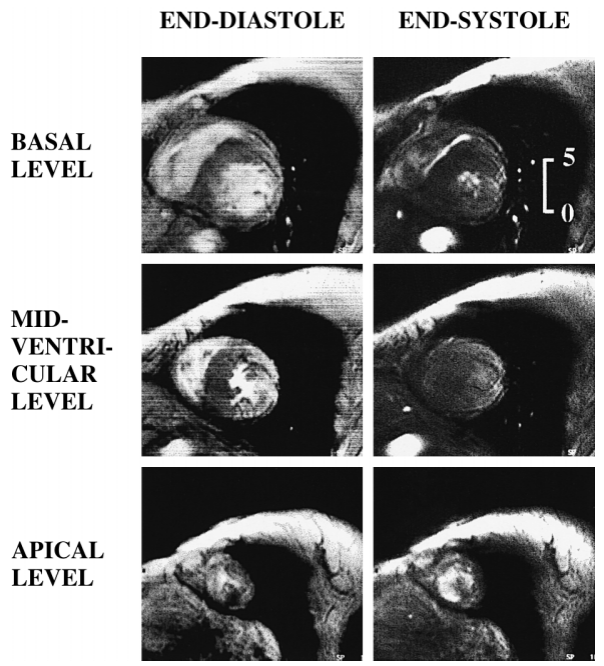

Fig 3. Cardiac magnetic resonance imaging (MRI) in the vertical long axis view at end-systolic phase (panel A), and the short axis views (panel B), depicting the apical aneurysm $(\rightarrow)$ and midventricular obstruction, which separated the left ventricular cavity into 2 chambers. 

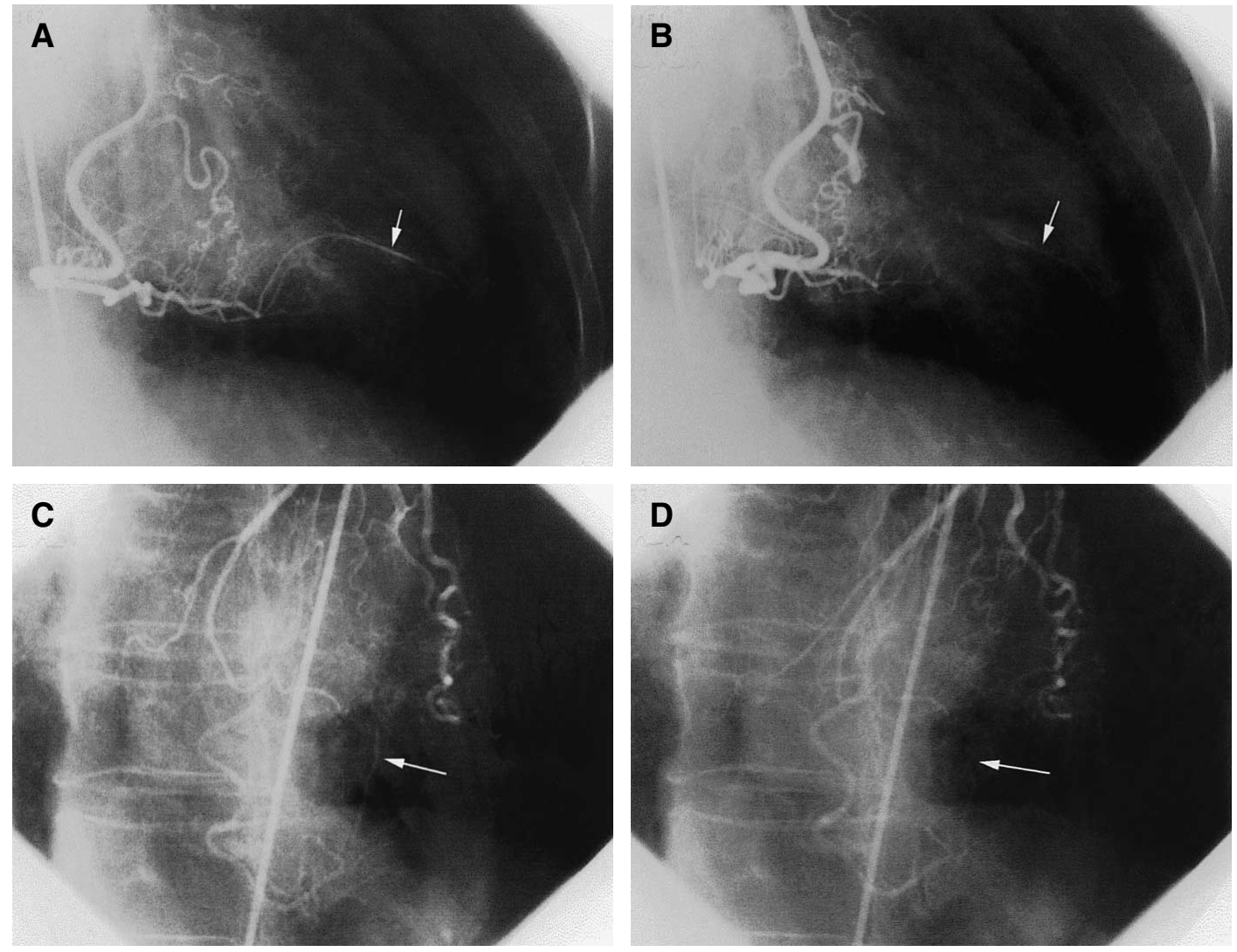

Fig 4. Coronary angiograms showing the compression of the aberrant coronary arteries (as marked) from the right (panels $\mathrm{A}, \mathrm{B}$ ) and left (panels C,D) coronary arteries.

chamber to the subaortic low-pressure chamber in the left ventricle. Left ventriculography revealed dyskinesis in the apical wall and a midventricular obstruction that separated the left ventricular cavity into 2 chambers. Coronary angiograms showed no narrowing of the extramural coronary arteries, but there was compression of the aberrant arteries, originating from the distal left anterior descending artery or from the posterior descending artery, which arteries appeared to feed the hypertrophic portion of the left ventricular wall (Fig 3).

Stress thallium-201 $\left({ }^{201} \mathrm{Tl}\right)$ myocardial imaging showed a persistent severe defect, suggesting myocardial scarring in the apical wall (Fig 4), but did not show any evidence of severe septal hypertrophy. After oral carvedilol (2.5 $\mathrm{mg}$ /day) was started for treatment of the intraventricular pressure gradient and ventricular arrhythmia, she was discharged from the hospital and did not have recurrence of symptoms during a follow-up period of 18 months.

\section{Discussion}

The present case of an elderly female patient with HCM with a midventricular obstruction, causing a severe intraventricular pressure gradient, and an apical aneurysm in the left ventricle, also showed compression of aberrant coronary arteries feeding the hypertrophic portion of the left ventricular wall. HCM has been reported to have some variants with atypical features in elderly women and one of them is characterized by severe concentric left ventricular hypertrophy with a history of systemic hypertension and abnormal left ventricular diastolic function; 12 another is

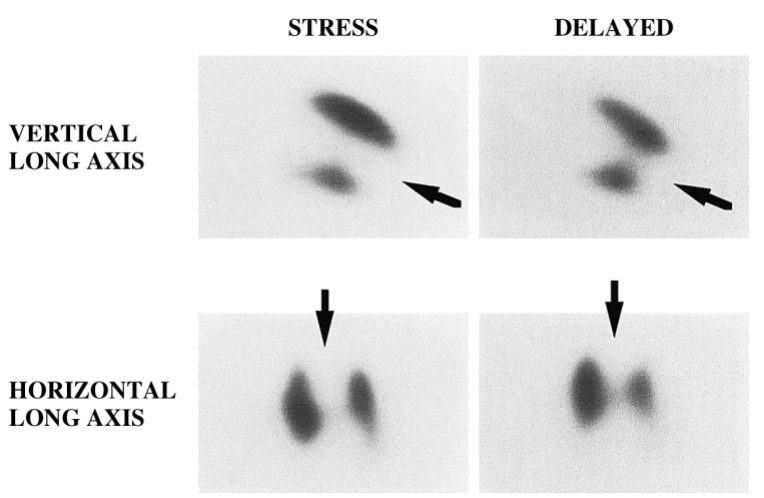

Fig 5. Stress thallium-201 myocardial imaging. A persistent severe defect was observed in the apex (as marked) on both the initial (left panels) and delayed images (right panels).

characterized by obstructive HCM with submitral annular calcification. ${ }^{13}$ However, the present case did not have either of these 2 variations.

Midventricular obstruction is a rare form of HCM, which can be complicated with apical myocardial infarction and normal extramural coronary arteries. Fighali et al showed that segmental or generalized left ventricular hypokinesis could develop in patients with HCM and midventricular obliteration without a fixed coronary artery obstruction? and they speculated that a possible cause of such hypokinesis was that the large pressure gradient at the midventricular level might lead to further compensatory 
apical hypertrophy, which could in turn make the flow obstruction more severe and the pressure in the apical chamber higher. The pressure overload on the apical wall might eventually lead to myocardial dysfunction with dilation of the apical chamber. This hypothesis is consistent with the present case in which the peak pressure of the apical chamber exceeded $185 \mathrm{mmHg}$ and Doppler echocardiography revealed paradoxical jet flow from the apical aneurysm to the left ventricular outflow during early diastole 14 $^{14}$

Other possible causes of myocardial ischemia in HCM include small-vessel disease with decreased vasodilator capacity, septal perforator artery compression, myocardial bridging, decreased coronary perfusion pressure, embolization of a major coronary artery branch, coronary artery spasm, oxygen supply/demand mismatch, and decreased capillary myocardial fiber ratio $3,15-17$ In the present case, myocardial ischemia was most likely caused by the compression of the aberrant coronary arteries apparently feeding the hypertrophic portion of the left ventricular wall. Lack of contrast accumulation in the hypertrophic portion and a persistent severe defect in the apical wall on stress ${ }^{201} \mathrm{Tl}$ myocardial imaging are also consistent with myocardial ischemia or infarction. Coronary abnormality (aberrant intramural coronary arteries and compression) combined with mechanical distress (intraventricular pressure gradient) is the most plausible cause of the apical myocardial infarction in the present case. Webb et al reported loss of precordial giant $\mathrm{T}$-wave negativity with formation of an apical aneurysm during a 17-year follow-up in a patient with apical hypertrophy and normal coronary arteries? Although the electrocardiogram of the present case was similar to the one that Webb et al reported (Fig 1), it only suggests the complication of apical myocardial aneurysm with left ventricular hypertrophy after loss of giant T-wave negativity.

Despite the normal appearance of the major extramural coronary arteries, abnormal intramural coronary arteries, with a reduced lumen and thickening of the vessel wall, are common in HCM, occurring in more than $80 \%$ of patients, 3,18 and may be intrinsic to the condition. Recently, the hamster BIO 14.6, a model of HCM, was shown to be caused by a 5 ' deletion of the $\delta$-sarcoglycan gene, ${ }^{19}$ suggesting that it may be involved in the pathogenesis of HCM. On the other hand, $\delta$-sarcoglycan knockout mice developed cardiomyopathy associated with vascular malfunction and irregularities of the coronary artery vasculature ${ }^{20}$ Thus, in the present case, it is possible that abnormalities of the coronary vasculature or aberrant intramural coronary arteries may have contributed to the genesis of the apical aneurysm.

Although mutations in the human essential myosin light chains (Met149Val) and regulatory light chains (Glu22Lys, Pro94Arg) have been recently shown to result in papillary muscle hypertrophy and subsequent midventricular obstruction, 21 these mutations have not been detected so far in the present case.

Management of HCM with midventricular obstruction is difficult and although ventricular septal myotomy-myectomy or dual-chamber (DDD) pacing is the treatment of choice for patients with severe outflow tract obstruction refractory to drug treatment 5,22 its role in this subgroup of patients with HCM is not yet established. Without other predictors of outcome, except for an outflow gradient no less than $30 \mathrm{mmHg}{ }^{23,24}$ we began treatment with a $\beta$ - blocker, although some HCM centers favor verapamil or class Ia antiarrhythmic agents. ${ }^{25}$ This case needs close observation because transition to the dilated phase has been reported in a patient with HCM with midventricular obstruction ${ }^{26}$

In conclusion, the present case is a rare combination of clinical features and suggests that the coronary vasculature abnormality in HCM, if complicated with mechanical distress such as midventricular obstruction, may result in an apical aneurysm.

\section{References}

1. Maron BJ, Bonow RO, Cannon RO, Leon MB, Epstein S: Hypertrophic cardiomyopathy: Interrelations of clinical manifestations, pathophysiology, and therapy. N Engl J Med 1987; 316: 780-789, $844-852$

2. Braunwald E: Hypertrophic cardiomyopathy: Continued progress. $N$ Engl J Med 1989; 320: 800-802

3. Maron BJ: Hypertrophic cardiomyopathy. Curr Probl Cardiol 1993; 18: $642-693$

4. Thierfelder L, Watkins H, MacRae C, Lamas R, McKenna W, Vosberg HP, et al: $\mathbf{\alpha}$-tropomyosin and cardiac troponin T mutations cause familial hypertrophic cardiomyopathy: A disease of the sarcomere. Cell 1994; 77: 701-712

5. Wigle ED, Rakowski H, Kimball BP, Williams WG: Hypertrophic cardiomyopathy: Clinical spectrum and treatment. Circulation 1995; 92: $1680-1692$

6. Wigle ED, Sasson Z, Henderson MA, Ruddy TD, Fulop J, Rakowski $\mathrm{H}$, et al: Hypertrophic cardiomyopathy: The importance of the site and the extent of hypertrophy: A review. Prog Cardiovasc Dis 1985; 28: $1-83$

7. Fighali S, Krajcer Z, Edelman S, Leachman RD: Progression of hypertrophic cardiomyopathy into a hypokinetic left ventricle: Higher incidence in patients with midventricular obstruction. $J \mathrm{Am}$ Coll Cardiol 1987; 9: 288-294

8. Ishiwata S, Nishiyama S, Nakanishi S, Sekie A: Two types of left ventricular wall motion abnormalities with distinct clinical features in patients with hypertrophic cardiomyopathy. Eur Heart J 1993; 14: $1629-1639$

9. Webb JG, Sasson Z, Pakowski H, Liu P, Wigle ED: Apical hypertrophic cardiomyopathy: Clinical follow-up and diagnostic correlates. J Am Coll Cardiol 1990; 15: 83-90

10. Akutsu Y, Shinozuka A, Huang T-Y, Watanabe T, Yamada T, Yamanaka $\mathrm{H}$, et al: Hypertrophic cardiomyopathy with apical left ventricular aneurysm. Jpn Circ J 1998; 62: 127-131

11. Nakamura T, Matsubara K, Furukawa K, Azuma A, Sugihara H, Katsume H, et al: Diastolic paradoxic jet flow in patients with hypertrophic cardiomyopathy: Evidence of concealed apical asynergy with cavity obliteration. J Am Coll Cardiol 1992; 19: 516-524

12. Topol EJ, Trail TA, Fortuin NJ: Hypertensive hypertrophic cardiomyopathy of the elderly. N Engl J Med 1985; 312: 277-283

13. Lewis JF, Maron BJ: Elderly patients with hypertrophic cardiomyopathy: A subset with distinctive left ventricular morphology and progressive clinical course late in life. J Am Coll Cardiol 1989; 13: $36-45$

14. Nakamura T, Matsubara K, Furukawa K, Kitamura H, Azuma A, Sugihara H, et al: Apical sequestration in hypertrophic cardiomyopathy: Its clinical features and pathophysiology. J Cardiol 1991; 21: 361-374

15. Maron BJ, Epstein SE, Roberts WC: Hypertrophic cardiomyopathy and transmural myocardial infarction without significant atherosclerosis of the extramural coronary arteries. Am J Cardiol 1979; 43: $1086-1102$

16. Cannon RO 3rd, Rosing DR, Maron BJ, Leon MB, Bonow RO, Watson RM, et al: Myocardial ischemia in patients with hypertrophic cardiomyopathy: Contribution of inadequate vasodilator reserve and elevated left ventricular filling pressures. Circulation 1985; 71: 234243

17. Tomochika Y, Tanaka N, Wasaki Y, Shimizu H, Hiro J, Takahashi $\mathrm{T}$, et al: Assessment of flow profile of left anterior descending coronary artery in hypertrophic cardiomyopathy by transesophageal pulsed Doppler echocardiography. Am J Cardiol 1993; 72: $1425-$ 1430

18. Louie EK, Edwards LC 3rd: Hypertrophic cardiomyopathy. Prog Cardiovasc Dis 1994; 36: 275-308

19. Sakamoto A, Abe M, Masaki T: Delineation of genomic deletion in 
cardiomyopathic hamster. FEBS Lett 1999; 447: 124-128

20. Coral-Vazquez R, Cohn RD, Moore SA, Hill JA, Weiss RM, Davisson RL, et al: Disruption of the sarcoglycan-sarcospan complex in vascular smooth muscle: A novel mechanism for cardiomyopathy and muscular dystrophy. Cell 1999; 98: 465-474

21. Poetter K, Jiang H, Hassanzadeh S, Master SR, Chang A, Dalakas $\mathrm{MC}$, et al: Mutation in either the essential or regulatory light chains of myosin are associated with a rare myopathy in human heart and skeletal muscle. Nat Genet 1996; 13: 63-69

22. Fananapazir L: Advances in molecular genetics and management of hypertrophic cardiomyopathy. JAMA 1999; 281: 1746-1752

23. Maron BJ: Hypertrophic cardiomyopathy. Lancet 1997; 350: $127-$
133

24. Maron BJ, Casey SA, Poliac LC, Gohman TE, Almquist AK, Dorothee MA: Clinical course of hypertrophic cardiomyopathy in a regional United States cohort. JAMA 1999; 282: 650-655

25. Ohtsuka T, Hamada M, Hara Y, Kuwahara T, Kodama K, Shigematsu Y, et al: An early systolic sound associated with midventricular obstruction in a patient with hypertrophic cardiomyopathy. Jpn Circ $J$ 1998; 62: 385-388

26. Kawai M, Kihara Y, Hasegawa K, Matsumori A, Sasayama S: Dilated phase of hypertrophic cardiomyopathy with mid-ventricular obstruction after 20-year follow-up. Jpn Circ J 2000; 64: 623-626 